\title{
CONFORTO/DESCONFORTO EM DOENTES INTERNADOS EM CLÍNICA PSIQUIÁTRICA
}

| João Apóstolo; Maria Antunes²; Aida Mendes³ Inês Castro $^{4}$ |

\section{RESUMO}

Considerando o conforto, de acordo com Kolcaba (1991; 2003), como um estado em que estão satisfeitas as necessidades humanas básicas relativamente ao alívio, tranquilidade e transcendência nos quatro contextos da experiência - físico, "psicoespiritual", sociocultural e ambiental -, verificámos que os doentes internados em psiquiatria clínica experienciam uma condição de desconforto relacionado com os sintomas que resultam da doença e do internamento em si mesmo.

Objectivo: Descrever as características do conforto/ desconforto de doentes internados em serviços de clínica psiquiátrica.

Método: Estudo descritivo com utilização da Escala para a Avaliação do Conforto em Doentes Internados em Serviços de Clínica Psiquiátrica (ECIP), tipo Likert com 5 pontos, baseada na estrutura conceptual de Kolcaba (2003). Amostra: 393 indivíduos, 215 mulheres e 178 homens, com idade média 41,26; DP 13,36 anos.

Resultados: No global da escala $27,48 \%$ dos doentes experienciaram um grau de desconforto elevado ou moderado. Foi nas dimensões transcendência e "psicoespiritual" que os doentes referiram maior desconforto com, respectivamente, $46,05 \%$ e $41,47 \%$.

Conclusão: Os doentes sentem maior desconforto relativamente à consciência de si, ao sentido de vida, auto-estima, auto-conceito, bem como no potencial para planear, controlar o seu destino e resolver os seus problemas. A suportar esta evidência existem resultados anteriores, com recurso a metodologia qualitativa (Apóstolo, 2009; 2010), revelando que estes doentes se sentem aprisionados na doença e limitados no desenvolvimento do seu projecto de vida. Assim, a intervenção deverá ser orientada, essencialmente, para estes aspectos.

\section{PALAVRAS-CHAVE: Conforto; avaliação; psiquia- tria; hospital}

\section{ABSTRACT}

In accordance with Kolcaba $(1991 ; 2003)$ we considered comfort as a state in which the basic human needs of relief, tranquility and transcendence are met in the four contexts of experience - physical, "psychospiritual", sociocultural and environmental - and verified that psychiatric inpatients experience discomfort related to the symptoms that result from their illness and from hospitalization.

Aim: To describe the characteristics of comfort/discomfort of inpatients in clinical psychiatric facilities.

Method: Descriptive study using the Psychiatric Inpatients Comfort Scale (PICS), 5 point Likert type, based on the conceptual structure of Kolcaba (2003). Sample: 393 individuals, 215 women and 178 men, mean age 41.26; SD 13.36. Previous studies have demonstrated the psychometric properties of PICS (Apóstolo et al., 2007). For the sample under analysis Cronbach's alfa of the total scale is 0.91 .

Results: In the total scale $27.48 \%$ of patients experience a high or moderate level of discomfort. The dimensions transcendence and "psychospiritual" were the ones in which patients referred higher discomfort with, respectively, $46.05 \%$ and $41.47 \%$.

Conclusion: Patients feel greater discomfort relating to the conscience of self, meaning of life, self-esteem, selfconcept, as well as the potential to make plans, control one's destiny and solve one's problems. There are previous results from qualitative studies (Apóstolo, 2009; 2010) that support this evidence having shown that patients feel imprisoned in their illness and limited in the development of their life project. In this way, interventions ought to be oriented towards these aspects.

\section{KEYWORDS: Comfort; assessment; psychiatry; hos- pital}

\section{INTRODUÇÃO}

Apesar de diferentes modelos de cuidados comunitários se terem desenvolvido devido à gravidade da doença mental e dos seus sintomas, é necessária, muitas vezes, intervenção especializada ao nível hospitalar, levando o doente e a família a suportar e a escolher esta opção como uma oportunidade para a reconstrução pessoal. $\mathrm{O}$ internamento, apesar de ser necessário para o restabelecimento da saúde, providenciando suporte técnico, ambiente controlado e seguro, pode também ser responsável pela separação da pessoa do seu contexto e das suas circunstâncias gerando, a par com a doença, sofrimento e desconforto. O conforto tem assumido um papel relevante na filosofia dos cuidados de enfermagem e é reconhecido como um resultado holístico que

\footnotetext{
1 RN, PhD; Professor adjunto - Escola Superior de Enfermagem de Coimbra, Portugal. Apartado 55, 3001-901, Coimbra, Portugal, apostolo@esenfc.pt

2 PhD; Professora Coordenadora - Escola Superior de Enfermagem de Coimbra, tcalvario@esenfc.pt

3 RN, PhD; Professora Coordenadora - Escola Superior de Enfermagem de Coimbra, amendes@esenfc.pt

4 Bolseira de Integração na Investigação (BII) da Unidade de Investigação em Ciências da Saúde: domínio de Enfermagem (UICISA-dE), castrines@gmail.com

Submetido em: 30-03-2012 - Aceite em 30-05-2012

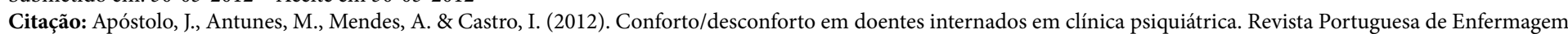


diz respeito às respostas da pessoa como um todo (Kolcaba, 2003). A teoria do conforto tem sido usada para explicar e predizer fenómenos de interesse relativos às respostas humanas no percurso saúde-doença, contribuindo também para uma avaliação fundamentada dos cuidados e medição dos resultados das intervenções.

Neste contexto, o conforto, seja ele considerado como um processo, o de confortar, ou como um resultado, o das intervenções, é um conceito nobre e subjacente à intervenção dos técnicos de saúde que intervêm no processo de saúde-doença e nas respostas humanas a este processo.

A estrutura conceptual que suporta este estudo deriva da teoria de médio alcance do conforto de Kolcaba (1991). A autora define conforto como "a satisfação (activa, passiva ou cooperativa) das necessidades humanas básicas de alívio, tranquilidade e transcendência que emergem de situações causadoras de stresse" (Kolcaba, 1994, p. 1178).

O termo conforto deriva do latim confortare, que significa restituir as forças físicas, o vigor e a energia; tornar forte, fortalecer, revigorar (Instituto de Lexicologia e Lexicografia da Academia das Ciências de Lisboa, 2001). À luz destes significados, o conceito conforto poderia ser mal interpretado e considerado demasiado vago para ser estudado, e medido, como componente da saúde e bem-estar do indivíduo. Para além disso, o conforto terá um significado muito mais complexo e diferenciado, do que aquele que depreendemos da etimologia. A teoria de enfermagem tem demonstrado que é uma dimensão ou uma componente de processos, de experiências e de conceitos dinâmicos tais como, a qualidade de vida, a esperança, o controlo e a tomada de decisão. Assim, o controlo e ausência de dor é considerado, muitas vezes, sinónimo de conforto, enquanto a presença e sensação de dor descreve, variadas vezes, o sentido da palavra desconforto. Ao mesmo tempo, o desconforto é tipicamente relatado como a não satisfação de algumas necessidades que, quando satisfeitas, resultam na experiência de conforto. No entanto, o estado de conforto não pressupõe a ausência total do desconforto, uma vez que a sensibilidade para o desconforto é relativa, variando de pessoa para pessoa (Kolcaba, 2003).

Kolcaba \& Kolcaba (1991) consideram os seguintes significados do conforto.

O primeiro significado diz respeito ao conforto como uma causa de alívio do estado de desconforto ou uma causa do estado de conforto.

O segundo significado remete para o conforto como um estado de tranquilidade e de pacífico contentamento.
Assim, o conforto como uma causa (primeiro significado) produz, supostamente, o conforto como um efeito (segundo significado).

Certas condições de vida, como preocupações, dor ou sofrimento implicam uma ausência do estado de conforto. A existência destas condições é denominada de desconforto, cujo estado resultante é contrário ao estado de conforto. Contudo, é de salientar que o estado de conforto pode existir sem que antes tenha existido desconforto (estado de tranquilidade). Quando o desconforto não pode ser evitado, é comum atenuá-lo com confortos adicionais, aliviando o desconforto.

O terceiro significado refere-se ao conforto como sendo o alívio do desconforto, o que pode ser explicado através dos dois significados anteriores. A causa de alívio é explicada pelo primeiro significado, ao passo que o estado de conforto é especificado pelo segundo. Embora o alívio em si mesmo seja apelidado de conforto, pode não ser equivalente ao estado de conforto, uma vez que este pode ser incompleto, parcial ou temporário, dado que pode constituir o alívio de apenas um de muitos desconfortos. Pode ainda ser parcial porque se atingiu apenas um grau de alívio temporário, uma vez que este pode perdurar apenas até o desconforto surgir novamente. Deste modo, o estado de conforto tem subjacente a inexistência de qualquer desconforto e um grau elevado e duradouro de alívio do desconforto. O quarto significado aponta o conforto como o que torna a vida fácil e agradável. Este significado refere-se à função de maximizar o prazer e, por esta razão, está fora do âmbito dos cuidados de enfermagem.

Os autores apontam ainda para um quinto e sexto significados do conforto que remetem para a etimologia da palavra latina confortare, que significa "fortalecer grandemente". O quinto significado pode traduzir força, encorajamento, incitação, ajuda, socorro, suporte e contentamento. O conforto pode ainda indicar (sexto significado) influência vigorosa ou refrescamento físico. Estes significados referem-se a causas de renovação, amplificações de poder, estados de espírito positivos e preparação para a acção. Neste sentido, estarão na origem do conforto as coisas que fortalecem e encorajam, suportam e/ou fisicamente refrescam ou vigorizam uma pessoa.

Em síntese, o conforto é um estado em que estão satisfeitas as necessidades básicas relativamente ao alívio, tranquilidade e transcendência (Kolcaba, 1991; 1994; 2003). Estes três estados - alívio, tranquilidade e transcendência - são assim operacionalizados na teoria de Kolcaba. O alívio é o estado em que uma necessidade foi satisfeita, sendo necessário para que a pessoa restabeleça 
o seu funcionamento habitual; o segundo estado, tranquilidade, é um estado de calma ou de satisfação, necessário para um desempenho eficiente; o terceiro estado, transcendência, é o estado no qual cada pessoa sente que tem competências ou potencial para planear, controlar o seu destino e resolver os seus problemas. Este tipo de conforto é também chamado de renovação. Estes três estados de conforto desenvolvem-se em quatro contextos: físico, "psicoespiritual", sociocultural e ambiental. O contexto físico diz respeito às sensações corporais; o contexto sociocultural às relações interpessoais, familiares e sociais; o contexto "psicoespiritual" à consciência de si, incluindo a auto-estima e o autoconceito, a sexualidade e o sentido de vida, podendo também envolver uma relação com uma ordem ou ser superior; e o contexto ambiental envolve aspectos como a luz, barulho, equipamento (mobiliário), cor, temperatura e elementos naturais ou artificiais do meio (Kolcaba, 1991; 2003).

Num diagrama, a intercepção destes três estados com os quatro contextos em que ocorrem definem 12 células. O conjunto destas células representa a forma total do conforto holístico, sendo que cada atributo de conforto está representado em cada uma das doze células da estrutura taxonómica. Cada célula reflecte a síntese dos significados das duas dimensões (estados e contextos), representando cada uma per si, uma faceta diferente do conforto. Por serem interdependentes, uma mudança numa delas provoca uma mudança nas outras (Kolcaba, 2003).

Figura 1: Estrutura conceptual do conforto (Kolcaba, 1991)

\begin{tabular}{|c|l|l|l|}
\hline Contextos & Alívio & Tranquilidade & Transcendência \\
\hline Físico & $\begin{array}{l}\text { Alívio } \\
\text { Físico }\end{array}$ & $\begin{array}{l}\text { Tranquilidade } \\
\text { Física }\end{array}$ & $\begin{array}{l}\text { Transcendência } \\
\text { Física }\end{array}$ \\
\hline "Psicoespiritual" & $\begin{array}{l}\text { Alívio } \\
\text { Psicoespiritual }\end{array}$ & $\begin{array}{l}\text { Tranquilidade } \\
\text { Psicoespiritual }\end{array}$ & $\begin{array}{l}\text { Transcendência } \\
\text { Psicoespiritual }\end{array}$ \\
\hline Ambiental & $\begin{array}{l}\text { Alívio } \\
\text { Ambiental }\end{array}$ & $\begin{array}{l}\text { Tranquilidade } \\
\text { Ambiental }\end{array}$ & $\begin{array}{l}\text { Transcendência } \\
\text { Ambiental }\end{array}$ \\
\hline Sociocultural & $\begin{array}{l}\text { Alívio } \\
\text { Sociocultural }\end{array}$ & $\begin{array}{l}\text { Tranquilidade } \\
\text { Sociocultural }\end{array}$ & $\begin{array}{l}\text { Transcendência } \\
\text { Sociocultural }\end{array}$ \\
\hline
\end{tabular}

\section{METODOLOGIA}

Foi desenvolvido um estudo de tipo quantitativo descritivo, com as seguintes questões de investigação:

Q1: Quais os níveis de conforto/desconforto experienciados pelos doentes internados em serviços de clínica psiquiátrica?

Q2: Qual é a severidade do desconforto experienciado por estes doentes?

\section{Amostra}

A amostra foi constituída por 393 doentes internados em serviços de clínica de três hospitais psiquiátricos da região de Coimbra e um do Porto, 215 mulheres e 178 homens, com idade média 41,26; DP 13,36 anos. $48,60 \%$ eram casados ou viviam em união de facto, $34,86 \%$ solteiros, $13,49 \%$ divorciados e $3,05 \%$ viúvos. Relativamente à escolaridade (em anos), 27,74\% tinham $4,40,20 \%$ entre 5 e $9,16,28 \%$ entre 10 e 12 e $15,78 \%$ ensino superior.

Relativamente aos diagnósticos psiquiátricos, 50,89\% dos doentes foram incluídos no grupo diagnóstico perturbações do humor; $31,81 \%$ no grupo esquizofrenia, perturbações esquizotípicas e perturbações delirantes e $17,30 \%$ no grupo perturbações neuróticas, perturbações relacionadas com o stresse e perturbações somatoformes.

\section{Instrumento}

O instrumento de recolha de dados foi constituído por um conjunto de questões sócio-demográficas e clínicas e pela Escala de avaliação do conforto em doentes internados em serviços de clínica psiquiátrica (ECIP) (Apóstolo et al., 2007).

A ECIP é um instrumento baseado no "modelo operacional do conforto" de Kolcaba (2003), constituído por 42 itens, que se propõe medir o conforto de três estados (Alívio, Tranquilidade e Transcendência) em quatro contextos (Físico, Psico-espiritual, Sócio-cultural e Ambiental). É uma escala do tipo Likert, de cinco pontos, que correspondem a cinco âncoras: 1 - não corresponde nada ao que se passa comigo; 2 - corresponde pouco ao que se passa comigo; 3 - corresponde bastante ao que se passa comigo; 4 - corresponde muito ao que se passa comigo e 5 - corresponde totalmente ao que se passa comigo.

Estudos prévios demonstraram as qualidades psicométricas da ECIP (Apóstolo et al., 2007; Apóstolo, 2010). $\mathrm{Na}$ amostra em estudo o alfa de Cronbach para o total da escala e dimensões oscilou entre 0,72 e 0,91 .

O grau de conforto/desconforto foi calculado, tendo como critério as referidas âncoras e considerando-se as seguintes pontuações médias para cada dimensão e total: de 1 a 1,99 - desconforto elevado; de 2 a 2,99 desconforto moderado; de 3 a 3,99 - conforto moderado; de 4 a 5 - conforto elevado.

\section{Procedimentos}

O estudo foi aprovado pelas Comissões de Ética dos quatro hospitais psiquiátricos em causa. Os doentes responderam aos questionários após assinarem o consentimento informado. Nos casos que não apresentavam 
limitações na interpretação, os questionários foram auto-administrados. Aqueles que mostraram alguma limitação contaram com a ajuda dos investigadores e colaboradores. Os dados foram colhidos em 2006 e 2007.

Para analisar os dados foram utilizadas medidas estatísticas, intervalo de confiança para a média e frequências.

\section{Resultados}

Níveis de conforto/desconforto:

As respostas aos 42 itens da ECIP oscilam entre o valor máximo (5) e mínimo (1). Analisando as pontuações médias obtidas, verificamos que os doentes apresentam maiores níveis de desconforto nos itens: 4 , que avalia um sentimento de satisfação com o mundo (tranquilidade "psicoespiritual"); 5, que avalia satisfação com as relações íntimas (transcendência "psicoespiritual"); 9, que avalia sentimento de liberdade (transcendência "psicoespiritual”); 29, que avalia força física (transcendência física); 30, que avalia capacidade para resolver os problemas económicos (transcendência "psicoespiritual"); 32 , que avalia necessidade de melhor informação sobre a doença (alívio social); e 40, que avalia relaxamento corporal (tranquilidade física). Os valores médios nas respostas a estes itens oscilam entre 2,68 e 2,92, inferiores ao ponto mediano da escala.

Os itens onde os doentes apresentam maior conforto são: o 3, que avalia sentimento de rejeição familiar (alívio sociocultural); 11, que avalia confiança nos profissionais de saúde (tranquilidade social); 17, que avalia capacidade para colaborar no tratamento (transcendência "psicoespiritual"); 19, que avalia sentimento de protecção no contexto terapêutico (tranquilidade ambiental); e 37, que avalia náuseas ou enjoo (alívio físico). Os valores médios nas respostas a estes itens oscilam entre 3,81 e 4,35 .

Dimensões em que é percepcionado maior conforto/ desconforto:

Ao analisar o Quadro 1 podemos verificar que os doentes percepcionam um nível médio de conforto global de 3,40, acima do nível médio da escala, IC 95\% [3,34; $3,46]$. Observa-se uma tendência semelhante relativamente aos vários estados e contextos, cujos valores médios oscilam entre 3,16 (transcendência), IC 95\% [3,07; 3,25] e 3,60 (alívio), IC 95\% [3,53; 3,67].

É na transcendência que se verifica um nível médio de conforto mais baixo $(3,16)$, mas esta é, também, a dimensão onde o intervalo de confiança tem maior amplitude.

Quadro 1 - Valores mínimos, máximos, médios e de dispersão das dimensões do conforto/desconforto de doentes internados em serviços de clínica psiquiátrica $(\mathrm{n}=393)$.

\begin{tabular}{|c|c|c|c|c|c|}
\hline Conforto/Desconforto & Min. & Máx. & Média & DP & IC 95 \% \\
\hline Alívio & 1,00 & 5,00 & 3,60 & 0,71 & 3,$53 ; 3,67$ \\
\hline Tranquilidade & 1,00 & 5,00 & 3,50 & 0,68 & 3,$43 ; 3,57$ \\
\hline Transcendência & 1,00 & 4,94 & 3,16 & 0,88 & 3,$07 ; 3,25$ \\
\hline Físico & 1,00 & 5,00 & 3,41 & 0,77 & 3,$33 ; 3,49$ \\
\hline "Psicoespiritual" & 1,00 & 5,00 & 3,27 & 0,79 & 3,$19 ; 3,35$ \\
\hline Sociocultural & 1,00 & 5,00 & 3,47 & 0,74 & 3,$40 ; 3,54$ \\
\hline Ambiental & 1,00 & 5,00 & 3,56 & 0,69 & 3,$49 ; 3,63$ \\
\hline Conforto global & 1,00 & 4,86 & 3,40 & 0,61 & 3,$34 ; 3,46$ \\
\hline
\end{tabular}

Severidade do desconforto:

No que respeita à severidade do desconforto podemos verificar (Tabela 1) que 27,48\% dos doentes experienciam um grau de desconforto global elevado ou moderado. É no estado de transcendência e no contexto "psicoespiritual" que os doentes percepcionam maior desconforto, com respectivamente $46,05 \%$ e $41,47 \%$ daqueles a apresentarem um grau elevado ou moderado.

Tabela 1 - Severidade do desconforto global, "psicoespiritual” e transcendência de doentes internados em serviços de clínica psiquiátrica (n=393).

\begin{tabular}{|l|c|c|c|c|c|c|}
\hline \multirow{2}{*}{$\begin{array}{c}\text { Severidade do } \\
\text { desconforto }\end{array}$} & \multicolumn{2}{|c|}{ Global } & \multicolumn{2}{c|}{ "Psicoespiritual" } & \multicolumn{2}{c|}{ Transcendência } \\
\cline { 2 - 8 } $\mathrm{n}^{\mathrm{o}}$ & $\%$ & $\mathrm{n}^{\circ}$ & $\%$ & $\mathrm{n}^{\circ}$ & $\%$ \\
\hline Desconforto elevado & 6 & 1,53 & 23 & 5,85 & 43 & 10,94 \\
\hline Desconforto moderado & 102 & 25,95 & 140 & 35,62 & 138 & 35,11 \\
\hline Conforto moderado & 215 & 54,71 & 154 & 39,19 & 140 & 35,62 \\
\hline Conforto elevado & 70 & 17,81 & 76 & 19,34 & 72 & 18,32 \\
\hline
\end{tabular}

Quanto às restantes dimensões é percepcionado um grau elevado ou moderado de desconforto relativamente ao alívio por $26,40 \%$ dos doentes; à tranquilidade por $25,19 \%$ dos doentes; ao conforto físico por 32,06\% dos doentes; ao conforto ambiental por $24,69 \%$ dos doentes e ao conforto sociocultural por $30,02 \%$ dos doentes.

\section{DISCUSSÃO E CONCLUSÕES}

Verificamos que é nas dimensões "psicoespiritual" e transcendência que os doentes apresentam maior desconforto. É nestas dimensões que se verificam valores médios mais baixos e é também nestas que uma maior percentagem de doentes sente mais desconforto, sendo o maior valor atinente ao estado de transcendência, com cerca de $46 \%$ dos doentes a experienciar desconforto elevado ou moderado.

Estas duas dimensões traduzem a consciência de si, o sentido da vida, a auto-estima, o auto-conceito, bem como o potencial para planear, controlar o seu destino e resolver os seus problemas. São dimensões que definem aspectos essenciais da condição humana. O ser humano é um sujeito auto-reflexivo, que se interroga acerca de si, dos outros e do mundo, que procura a todo o instante conferir significados às suas próprias experiências e ao 
mundo que o rodeia. A capacidade de encontrar respostas coerentes e positivas nesta sua actividade mental é condição essencial da saúde mental. A doença mental e o internamento hospitalar interferem, acima de tudo, com esta condição.

A suportar esta evidência estão resultados anteriores, com recurso a metodologia qualitativa (Apóstolo, 2009; 2010), revelando que estes doentes se sentem aprisionados na doença e limitados no desenvolvimento do seu projecto de vida. De acordo com o autor, o hospital é percebido, simultaneamente, como um refúgio, um espaço de liberdade, e também como um lugar onde a liberdade está limitada pelo processo de doença, pelo sofrimento, desconforto e mal-estar inerentes à hospitalização. $\mathrm{O}$ desconforto é essencialmente provocado pelo aprisionamento na doença e, em oposição, considera-se que o doente psiquiátrico se sente confortável quando não sente que o seu projecto de vida está posto em causa, quando deixa de se sentir constrangido, encarcerado.

Para além destas dimensões, convém referir que uma percentagem substancial de doentes, entre 25 e 32\%, experienciam um grau de desconforto elevado ou moderado nos restantes estados e contextos. De facto, o desconforto físico é experienciado por cerca de $32 \%$ dos doentes, sendo um importante aspecto a considerar na concepção dos cuidados de enfermagem em contexto psiquiátrico e indicando a importância do investimento terapêutico nesta dimensão.

De referir ainda dois dos itens da ECIP que apresentam valores médios de respostas mais elevados e que avaliam a confiança dos doentes nos profissionais de saúde e a protecção sentida no contexto terapêutico. Estes vêm confirmar os resultados do já referido estudo qualitativo (Apóstolo, 2010), segundo o qual o ambiente terapêutico hospitalar é percebido pelos doentes como um espaço de refúgio, de protecção e de liberdade. De facto, a doença aprisiona e é fonte das limitações existenciais e de projectos futuros, de desconforto e de sofrimento e a hospitalização surge, assim, como uma esperança possibilitadora de reconstrução.

No processo de hospitalização, para além da intervenção farmacológica, têm sido proporcionadas outras intervenções aos doentes internados em serviços de clínica psiquiátrica, como o relaxamento progressivo e a utilização de imagens mentais positivas, com o objectivo de diminuir os níveis de ansiedade e de stresse e promover o conforto, embora não se procedendo a uma avaliação dos resultados dessas intervenções de forma sistemática.

A literatura realça diversos estudos relativos ao efeito positivo destas intervenções na diminuição dos níveis de ansiedade, stresse e depressão (McKinney et al., 1997; Suk \& Yoon, 2001; Sloman, 2002; Campbell-Gillies, 2004), mas esses estudos foram desenvolvidos em contexto não psiquiátrico.

Apóstolo (2010) desenvolveu um estudo quasi-experimental numa amostra de 60 sujeitos internados em serviços de clínica psiquiátrica de curta duração. Os indivíduos do grupo que foi sujeito à intervenção, quando comparados com os do grupo de controlo, apresentaram um aumento significativo do conforto e uma diminuição da depressão, da ansiedade e do stresse. Não se verificou efeito no conforto ambiental. A variância explicada pela intervenção oscilou entre 6 e $23 \%$.

Intervenções deste tipo, tendo demonstrado um efeito que consideramos substancial no aumento do conforto e na diminuição dos níveis de ansiedade, depressão e stresse, poderão ser utilizadas como intervenções de enfermagem nos doentes internados em clínica psiquiátrica. Sendo uma intervenção autónoma de enfermagem, pode ser incluída no plano de cuidados dos doentes. Os riscos desta intervenção, desde que clinicamente acompanhada, são inexistentes, prevendo-se uma relação custo-benefício positiva.

\section{REFERÊNCIAS BIBLIOGRÁFICAS}

Academia das Ciências de Lisboa. Instituto de Lexicologia e Lexicografia (2001). Dicionário da língua portuguesa contemporânea. Lisboa: Editorial Verbo.

Apóstolo, J. L. A. (2009). Vivências do conforto-desconforto nos doentes internados em serviços de clínica psiquiátrica. Revista Portuguesa de Enfermagem de Saúde Mental, 2, 10-17.

Apóstolo, J. L. A. (2010). O conforto pelas imagens mentais na depressão ansiedade e stresse. Coimbra: Imprensa da Universidade de Coimbra.

Apóstolo, J. L. A., Kolcaba, K., Azeredo, Z. A., Antunes, M. T. C., \& Mendes, A. C. (2007). Avaliação das qualidades psicométricas da escala de avaliação do conforto em doentes psiquiátricos. Psychologica, 44, 489-504.

Campbell-Gillies, L. (2004). Guided imagery as treatment for anxiety and depression in breast cancer patients: A pilot study [On line]. Dissertação de mestrado. Johannesburg, SA: Rand Afrikaans University. [Consult. 8-2-07]. Disponível: http://etd.rau.ac.za/theses/ available/etd-10062004-095533/restricted/GIreviseddissert2003130304.pdf.

Kolcaba, K. Y. (1991). A taxonomic structure for the concept comfort. Image, 23 (4), 237-240. 
Kolcaba, K. Y. (1994). A theory of holistic comfort for nursing. Journal of Advanced Nursing, 19 (6), 11781184.

Kolcaba, K. Y. (2001). Evolution of the mid range theory of comfort for outcomes research. Nursing Outlook, 49 (2), 86-92.

Kolcaba, K. Y. (2003). Comfort theory and practice. A vision for holistic health care and research. New York: Springer.

Kolcaba, K. Y. (2007). The comfort line (On line). [Consult. 8-4-07]. Disponível:

http://www.thecomfortline.com.

Kolcaba, K. Y. \& Kolcaba, R. J. (1991). An analysis of the concept of comfort. Journal of Advanced Nursing, 16 (11), 1301-1310.
McKinney, C. H., Antoni, M. H., Kumar, M., Times, F. C., \& McCabe, P. M. (1997). Effects of guided imagery and music (GIM) therapy on mood and cortisol in healthy adults. Health Psychology, 16 (4), 390-400.

Sloman, R. (2002). Relaxation and imagery for anxiety and depression control in community patients with advanced cancer. Cancer Nursing, 25 (6), 432-435.

Suk, M. H. \& Yoon, Y. M. (2001). Effects of guided imagery on stress of adolescents. Korean Journal of Child Health Nursing [On line], 7 (3), 359-370. [Consult. 208-2007].

Disponível:http://www.koreamed.org/SearchBasic. php?RID=201248\&DT $=1$. 